\title{
Reduced Fibrinogen Survival in Diabetes Mellitus
}

\author{
A REVERSIBLE PHENOMENON
}

\author{
Robert L. Jones and Charles M. Peterson, The Rockefeller University, \\ New York 10021
}

\begin{abstract}
A B S T R A C T Fibrinogen survival and turnover were examined in 15 adult-onset diabetic patients. ${ }^{125}$ Ilabeled fibrinogen was prepared from each patient during the period of poor carbohydrate control, or hyperglycemic period, and fibrinogen survival determined. Improved control was established in each patient and during this euglycemic period, fibrinogen survival was determined simultaneously with ${ }^{125}$ I-fibrinogen saved from the hyperglycemic period and ${ }^{131}$ I-labeled fibrinogen prepared from the patient during the euglycemic period. The results confirm reduced fibrinogen survival in hyperglycemic diabetic patients and demonstrate reversal of the fibrinogen abnormality when euglycemia is achieved. The results of the double-label experiments in the euglycemic period suggest that the fibrinogen molecule is not altered functionally and that an abnormal plasma or vascular environment is a more likely basis for reduced fibrinogen survival during hyperglycemia. Electrophoretic and chromatographic experiments demonstrated no gross chemical differences between the fibrinogens prepared from the hyperglycemic and euglycemic periods and normal fibrinogen. Fibrinogen survival gave a better correlation with serial glucose measurements than with correction of hemoglobin $A_{I c}$ levels indicating that the reduced fibrinogen survival noted in diabetics is a rapidly reversible phenomenon.

During the hyperglycemic period, pharmacological intervention with aspirin and dipyrimadole was attempted to examine the role of platelets in reduced fibrinogen survival. No significant change in fibrinogen survival was observed. Heparin infusion during hyperglycemia normalized the fibrinogen kinetics of hyperglycemic diabetic patients, suggesting that reduced fibrinogen survival during hyperglycemia is secondary to an effect on thrombin or one of its antagonists.
\end{abstract}

Received for publication 5 July 1978 and in revised form 17 November 1978.

\section{INTRODUCTION}

The major controversies in diabetes mellitus center around the relationship of glycemia or carbohydrate "control" to the numerous sequelae of the disease. It has been difficult to address this relationship because of the problems attendant in the definition of carbohydrate control. It is certainly possible to hospitalize patients and measure serial blood glucose as a means of characterizing short-term control. In addition, it is now possible to define control over periods of weeks to months through the use of minor fast hemoglobins, especially hemoglobin $A_{\mathrm{lc}}$. This glycohemoglobin (the 1-N-valyl-1-deoxy-fructose adduct of hemoglobin A) results from a postsynthetic modification of normal hemoglobin A and provides an accurate assessment of the mean blood glucose over the previous 3-4 wk in individuals who are not suffering from hemolytic disease (1). Several sequelae have now been shown to be reversible when strict definitions of control are applied. These "reversible sequelae" include disorders of erythrocyte survival, leukocyte function, platelet function, and hyperlipidemia $(2,3)$.

Of the numerous secondary sequelae of diabetes, vascular disease now accounts for the major morbidity and mortality resulting from the diabetic state. The etiology of the increased risk of vascular disease in patients with diabetes mellitus is not clear. Most studies have focused on lipid abnormalities as the primary concomitant or causative factor predisposing the diabetic to vascular lesions. Recent studies suggest that abnormalities of the hemostatic system may also be involved in the initiation or propagation of vascular lesions (4).

Fibrinogen survival has been noted to be decreased in patients with diabetes (5). The present study was designed to determine whether the decreased survival of fibrinogen in patients with diabetes mellitus was reversible. With various measures of diabetic control, 
the time-course of the reversibility was assessed. In addition the studies were designed to assess whether the abnormalities in fibrinogen kinetics were the result of a postsynthetic modification of a normal fibrinogen molecule analogous to hemoglobin $A_{\mathrm{Ic}}$ production or the consequence of a normal molecule circulating in an abnormal environment. Finally, because the latter proved to be true, an attempt was made to pharmacologically correct the decreased fibrinogen survival seen in diabetics through the use of heparin infusions and aspirin and dipyrimadole administration.

\section{METHODS}

Patients. 15 patients with hyperglycemia and adult-onset, nonketotic diabetes mellitus of varying durations were admitted to The Rockefeller University Clinical Research Center. The patients had various complications including neuropathy, vascular disease, retinopathy, and(or) nephropathy as summarized in Table I. Diabetic neuropathy was identified by physical examination and nerve conduction studies. Vascular disease was evaluated by clinical findings as well as electrocardiographic criteria. Retinopathy was documented by fluorescein angiography and nephropathy by creatinine clearance. Patients with severe renal insufficiency as defined in Table I were excluded from the study.

Each patient was determined to be hyperglycemic by fasting blood glucose, urine sugars, glucose brackets, and hemoglobin $A_{\text {lc }}$ determinations. The "glucose brackets" is the sum of blood glucose values drawn before and $1 \mathrm{~h}$ after each meal for $1 \mathrm{~d}$. This value represents the patient's ability to metabolize dietary challenges and has been shown to correlate well with 24 -h integrated glucose concentrations (6). The blood glucose was brought under control by insulin or oral hypoglycemic therapy (chlorpropamide, Pfizer Laboratories Div., Pfizer Inc., New York), and dietary regulation (American Diabetes Association, 40-45\% fat diet). Glycemia was also monitored by urine sugars, glucose brackets, and hemoglobin $A_{\mathrm{Ic}}$.

To examine the possible mechanisms behind reduced fibrinogen survival the following protocol was adapted. In the hyperglycemic period, fibrinogen survival was studied with the patient's fibrinogen labeled with ${ }^{125}$ I. After the achievement of optimum blood glucose levels (euglycemic period), fibrinogen survival was determined simultaneously for ${ }^{131}$ Ilabeled fibrinogen purified from the patient during the euglycemic period and ${ }^{125}$ I-labeled fibrinogen saved from the hyperglycemic period.

Fibrinogen survival studies. Fibrinogen survival was determined with radioiodinated autologous fibrinogen labeled by the method of Takeda (7). This technique involved

TABLE I

Clinical Manifestations of 12 Diabetic Patients Studied for Fibrinogen Survival

\begin{tabular}{|c|c|c|c|c|c|c|c|}
\hline \multirow[b]{2}{*}{ Patient } & \multirow[b]{2}{*}{ Sex/age } & \multirow{2}{*}{$\begin{array}{l}\text { Duration of } \\
\text { diabetes } \\
\text { mellitus }\end{array}$} & \multirow[b]{2}{*}{ Therapy } & \multicolumn{4}{|c|}{ Complications $\S$} \\
\hline & & & & Vascular & Retinopathy & Neuropathy & Renal \\
\hline & $y r$ & $y r$ & & & & & \\
\hline 1 & $F / 56$ & 11 & Chlorpropamide & 0 & 0 & ++ & 0 \\
\hline 2 & M/63 & 11 & Insulin & ++ & ++ & ++ & + \\
\hline 3 & $\mathbf{M} / 61$ & 2 & Chlorpropamide & ++ & 0 & + & 0 \\
\hline 4 & $F / 50$ & 1 & Chlorpropamide & 0 & 0 & 0 & 0 \\
\hline 5 & $\mathbf{M} / 72$ & 25 & Insulin & + & ++ & ++ & + \\
\hline 6 & $\mathrm{~F} / 51$ & 4 & Chlorpropamide & 0 & 0 & + & 0 \\
\hline 7 & $\mathrm{M} / 58$ & 20 & Insulin & ++ & +++ & ++ & + \\
\hline 8 & $\mathrm{~F} / 29$ & 3 & Chlorpropamide & 0 & 0 & 0 & 0 \\
\hline 9 & M/59 & 2 & Chlorpropamide & 0 & 0 & ++ & 0 \\
\hline 10 & $\mathrm{M} / 51$ & 4 & Insulin & 0 & 0 & + & 0 \\
\hline 11 & $\mathrm{~F} / 57$ & 12 & Insulin & 0 & + & + & 0 \\
\hline 12 & $\mathrm{M} / 64$ & 5 & Insulin & + & + & +++ & 0 \\
\hline 13 & $\mathrm{M} / 77$ & 20 & Insulin & ++ & + & +++ & 0 \\
\hline 14 & $\mathrm{~F} / 52$ & 25 & Insulin & 0 & + & ++ & 0 \\
\hline 15 & $\mathrm{M} / 73$ & 26 & Insulin & + & ++ & ++ & 0 \\
\hline
\end{tabular}

* Vascular disease was rated by presence of electrocardiogram abnormalities, absence of peripheral pulses or present bruits over vessels, or history of angina or myocardial infarction, each $(+)$. Retinopathy was graded by fluorescein angiography: 0 , no diabetic changes; + , early change-scattered microaneurysms, rare retinal hemorrhages, or exudates; ++ , moderate change-numerous microaneurysms, retinal hemorrhages, hard and soft exudates, slight vitreous hemorrhage, early neovascularization within one disk diameter of the optic disk; +++ , severe change-extensive neovascularization, extensive fibroglial tissue proliferation, extensive vascular beading, extensive microaneurysm formation, marked retinal edema, lack of profusion of the capillaries over an extended area. Neuropathy was graded by: + , presence of abnormalities on surface electrode nerve conduction study; ++ , nerve conduction abnormality plus abnormal physical findings on sensory and motor exam; +++ , the above findings plus severe debilitation such as severely impaired ambulation. Renal involvement was judged by creatinine clearance $<60 \mathrm{ml} / \mathrm{min}$. No patients had a creatinine clearance $<20 \mathrm{ml} / \mathrm{min}$. 
the repeated precipitation of fibrinogen with $25 \%$ saturated ammonium sulfate solution followed by dissolution of the precipitate in $0.005 \mathrm{M}$ sodium citrate. Labeling was performed with ${ }^{125} \mathrm{I}$ - or ${ }^{131} \mathrm{I}-\mathrm{Cl}$ (monochloride) ([125 I]sodium iodide and $\left.{ }^{[31} \mathrm{I}\right]$ sodium iodide both reductant-free in $0.1 \mathrm{M} \mathrm{NaOH}$, New England Nuclear, Boston, Mass.). This was followed by reprecipitation with $25 \%$ saturated ammonium sulfate and removal of unbound radioiodine. After the solution was redissolved in $0.005 \mathrm{M}$ sodium citrate, it was cooled at $4^{\circ} \mathrm{C}$ for $1 \mathrm{~h}$ and centrifuged to remove cryoprecipitable material. The sample for injection was sterilized by filtration $(0.22 \mu \mathrm{m}$, Millipore Corp., Bedford, Mass.). Clottable radioactivity of the sample was affirmed to be $90 \%$ (range, 84-95\%) and the molar ratio of iodine to fibrinogen was always $<0.25$. Clottable radioactivity was followed over 4-5 d and analysis of the data points was performed with an exponential curve fitting program on a Hewlett-Packard HP-97 calculator (HewlettPackard Co., Palo Alto, Calif.). Fibrinogen survival was determined from the half-time disappearance divided by the natural logarithm of two. Fibrinogen turnover was calculated from the concentration of plasma fibrinogen divided by the survival time.

Pharmacological intervention. After an initial determination of fibrinogen survival, heparin therapy was begun in four patients still in the hyperglycemic period. Sodium heparin (Panheprin, Abbott Diagnostics, Diagnostic Products, North Chicago, Ill.) was administered as a continuous intravenous infusion by an intravenous drip or by syringe pump (Autosyringe, Nuclear Equipment Chemical Corp., Farmingdale, N. Y.) so that the activated partial thromboplastin time was maintained at 1.5 to 3 times control values. ${ }^{125}$ I-labeled autologous fibrinogen isolated during the hyperglycemic period was injected when the anticoagulant effect of heparin was achieved and clottable radioactivity followed until a halflife was again defined.

Aspirin $(330 \mathrm{mg})$ and dipyrimadole $(75 \mathrm{mg})$ were administered orally three times daily to four patients after the initial fibrinogen study in the hyperglycemic period. Elimination of the second wave of platelet aggregation was verified by aggregometer studies and fibrinogen survival studies were repeated with ${ }^{125}$ I-labeled fibrinogen isolated during hyperglycemia.

Clotting studies. Fibrinogen concentration was determined by a method based on the thrombin clottability of dilute plasma (8). Thrombin time was performed with bovine thrombin (100 National Institutes of Health $[\mathrm{NIH}] \mathrm{U} / \mathrm{ml}$ ) (Data-Fi, Dade Div., Miami, Fla). Prothrombin time and activated partial thromboplastin time were performed by standard methods (9) with Simplastin and Platelin (General Diagnostics Warner Lambert Company, Morris Plains, N. J.). Platelet aggregations studies with $70 \mu \mathrm{M}$ epinephrine final concentrations were performed by standard techniques (10) with a 2-channel aggregometer (Payton Associates, Buffalo, N. Y.) with a uniform siliconized stirring rod at $37^{\circ} \mathrm{C}$.

Biochemical determinations. Plasma glucose was measured by an AutoAnalyzer (Technicon Instruments Corp., Tarrytown, N. Y.). Serum triglycerides and cholesterol were measured by standard methods $(11,12)$. Urine sugar was measured semiquantitatively (on a scale of from 0 to $4+$ ) four times daily by standard methods (Clinitest, Ames Co., Elkhart, Ind.). Hemoglobin $A_{I c}$ was determined weekly on samples collected in EDTA by the column chromatographic method of Trivelli et al. (13) or by radioimmunoassay (14).

Electrophoretic and chromatographic studies. DEAEcellulose (DE52, Whatman Ltd., Maidstone, Kent, England) chromatography was performed at $4^{\circ} \mathrm{C}$ with a continuous Tris-phosphate gradient (15). Sephadex G-200 (Pharmacia Fine Chemicals, Piscataway, N. J.) column chromatography was performed at room temperature with a $0.2 \mathrm{M} \mathrm{NaCl}: 0.02 \mathrm{M}$ Tris buffer (1:5) pH 8.0 (16). For these experiments tracer amounts of ${ }^{125} \mathrm{I}$-labeled fibrinogen isolated during the hyperglycemic period and ${ }^{131}$ I-labeled fibrinogen samples isolated during the euglycemic period were mixed with $10-15 \mathrm{mg}$ of unlabeled fibrinogen purified in the same manner from a nondiabetic individual and dialyzed extensively against starting buffer. Samples from these columns were counted in a twochannel gamma counter (Packard Instrument Co. Inc., Downers Grove, Ill.) and absorbance at $280 \mathrm{~nm}$ measured.

Sodium dodecyl sulfate-polyacrylamide gel electrophoresis was performed on $8 \%$ gels with fibrinogen samples purified from the hyperglycemic and euglycemic periods, along with fibrinogen purified from a normal individual and commercially supplied human fibrinogen (Fraction 1, Sigma Chemical Co., St. Louis, Mo.) (17). Gels were stained with Coomassie brilliant blue. The concentration of fibrinogen in purified samples for chromatography and electrophoresis was determined spectrophotometrically at $280 \mathrm{~nm}$ with an $\left(\mathrm{A}_{280}^{1 \%}\right)$ of $13.9(18)$.

Statistics. Where applicable, values are expressed as the \pm 1 SD. The difference between means was assessed by the Student's $t$ test for paired data (19). Differences were termed significant if the $t$ value exceeded the $5 \%$ level.

\section{RESULTS}

Determinations of fibrinogen survival and turnover were performed in 15 patients during hyperglycemic and euglycemic periods. The interval between the end of the hyperglycemic period studies and the beginning of the euglycemic period varied between 1 and $9 \mathrm{wk}$ (mean, $2.5 \mathrm{wk}$ ). This interval was dependent upon the time required to achieve euglycemia as evidenced by absence of urine sugar and optimum glucose brackets. Table II documents glucose brackets, fibrinogen survival and turnover studies, and coagulation studies during the various periods of the study. Carbohydrate control, (as evidenced by glucose brackets) was significantly improved in all patients from a mean hyperglycemic value of 1514 I $490 \mathrm{mg} / 100 \mathrm{ml}$ to a mean euglycemic value of $710 \mathrm{I} 138 \mathrm{mg} / 100 \mathrm{ml}(P<0.001)$. Hemoglobin $A_{\text {Ic }}$ levels also showed a mean decrease of $1.7 \%$ of total hemoglobin from hyperglycemic to euglycemic periods. This decrease was not statistically significant, but the interval between studies was not sufficient for this measurement to reach a stable plateau during the control phase. Serum cholesterol (not shown) showed no significant change from hyperglycemic $(213 \pm 40 \mathrm{mg} / 100 \mathrm{ml})$ to euglycemic $(187 \pm 22$ $\mathrm{mg} / 100 \mathrm{ml}$ ) periods. Fasting serum triglycerides (not shown) also showed no significant change from during the two periods of the study $(135 \pm 55 \mathrm{mg} / 100 \mathrm{ml}$ to $124 \pm 42 \mathrm{mg} / 100 \mathrm{ml}$ ).

Prothrombin time, activated partial thromboplastin time, and thrombin time were in the normal range and did not change significantly from the hyperglycemic to euglycemic periods.

Plasma fibrinogen concentrations were elevated above normal range in 9 of the 15 patients studied. 
TABLE II

Results of Blood Glucose Regulation, Fibrinogen Survival and Turnover Studies, and Clotting Function Studies

\begin{tabular}{|c|c|c|c|c|c|c|c|}
\hline Patient & Period & $\begin{array}{l}\text { Glucose } \\
\text { brackets }\end{array}$ & $\mathrm{Hb} \mathrm{A_{ \textrm {lc } }}{ }^{*}$ & $\begin{array}{c}\text { Fibrinogen } \\
\text { concentrationt }\end{array}$ & $t^{1 / 2}$ & $\begin{array}{c}\text { Fibrinogen } \\
\text { survival }\end{array}$ & $\begin{array}{c}\text { Fibrinogen } \\
\text { turnover }\end{array}$ \\
\hline & & & $\%$ & $m g / m l$ & $d$ & $d$ & $\mathrm{mg} / \mathrm{mlld}$ \\
\hline \multirow[t]{4}{*}{1} & Hyperglycemia & 1,567 & 6.5 & 3.80 & 2.42 & 3.49 & 1.01 \\
\hline & Euglycemia & 825 & 3.6 & 4.00 & - & - & - \\
\hline & Hyperglycemia sample & & & & 3.67 & 5.29 & 0.76 \\
\hline & Euglycemia sample & & & & 3.50 & 5.05 & 0.79 \\
\hline \multirow[t]{4}{*}{2} & Hyperglycemia & 1,568 & 8.7 & 6.00 & 2.00 & 2.89 & 2.08 \\
\hline & Euglycemia & 890 & 5.4 & 5.25 & - & - & - \\
\hline & Hyperglycemia sample & & & & 3.62 & 5.23 & 1.01 \\
\hline & Euglycemia sample & & & & 3.87 & 5.59 & 0.94 \\
\hline \multirow[t]{4}{*}{3} & Hyperglycemia & 1,359 & 6.2 & 1.85 & 3.20 & 4.61 & 0.40 \\
\hline & Euglycemia & 758 & 5.2 & 1.80 & - & - & - \\
\hline & Hyperglycemia sample & & & & 3.96 & 5.70 & 0.31 \\
\hline & Euglycemia sample & & & & 3.96 & 5.70 & 0.31 \\
\hline \multirow[t]{4}{*}{4} & Hyperglycemia & 940 & 8.4 & 4.00 & 2.96 & 4.27 & 0.94 \\
\hline & Euglycemia & 568 & 8.0 & 4.20 & - & - & - \\
\hline & Hyperglycemia sample & & & & 4.50 & 6.49 & 0.65 \\
\hline & Euglycemia sample & & & & 4.17 & 6.01 & 0.69 \\
\hline \multirow[t]{4}{*}{5} & Hyperglycemia & 2,230 & 6.4 & 5.10 & 2.42 & 3.49 & 1.46 \\
\hline & Euglycemia & 617 & 5.9 & 4.60 & - & - & - \\
\hline & Hyperglycemia sample & & & & 3.17 & 4.57 & 1.00 \\
\hline & Euglycemia sample & & & & 3.29 & 4.75 & 0.97 \\
\hline \multirow[t]{4}{*}{6} & Hyperglycemia & 2,273 & 9.2 & 3.50 & 2.46 & 3.55 & 0.99 \\
\hline & Euglycemia & 843 & 8.1 & 3.80 & - & - & - \\
\hline & Hyperglycemia sample & & & & 3.46 & 4.99 & 0.76 \\
\hline & Euglycemia sample & & & & 3.41 & 4.93 & 0.77 \\
\hline \multirow[t]{4}{*}{7} & Hyperglycemia & 2,283 & 9.2 & 5.25 & 1.71 & 2.46 & 2.13 \\
\hline & Euglycemia & 940 & 7.5 & 2.25 & - & - & - \\
\hline & Hyperglycemia sample & & & & 3.25 & 4.68 & 0.48 \\
\hline & Euglycemia sample & & & & 3.08 & 4.45 & 0.50 \\
\hline \multirow[t]{4}{*}{8} & Hyperglycemia & 849 & 8.6 & 3.80 & 2.33 & 3.36 & 1.13 \\
\hline & Euglycemia & 653 & 6.0 & 4.00 & - & - & - \\
\hline & Hyperglycemia sample & & & & 3.04 & 4.39 & 0.91 \\
\hline & Euglycemia sample & & & & 3.04 & 4.39 & 0.91 \\
\hline \multirow[t]{4}{*}{9} & Hyperglycemia & 1,276 & 7.7 & 3.76 & 2.21 & 3.19 & 1.18 \\
\hline & Euglycemia & 653 & 5.3 & 3.70 & - & - & - \\
\hline & Hyperglycemia sample & & & & 2.92 & 4.21 & 0.88 \\
\hline & Euglycemia sample & & & & 3.06 & 4.41 & 0.84 \\
\hline \multirow[t]{4}{*}{10} & Hyperglycemia & 1,763 & 11.3 & 2.60 & 2.71 & 3.91 & 0.69 \\
\hline & Euglycemia & 692 & 9.7 & 2.45 & - & - & - \\
\hline & Hyperglycemia sample & & & & 3.67 & 5.30 & 0.46 \\
\hline & Euglycemia sample & & & & 3.50 & 5.05 & 0.49 \\
\hline \multirow[t]{4}{*}{11} & Hyperglycemia & 1,892 & 13.5 & 3.20 & 2.50 & 3.61 & 0.89 \\
\hline & Euglycemia & 520 & 12.2 & 3.20 & - & - & - \\
\hline & Hyperglycemia sample & & & & 3.58 & 5.17 & 0.62 \\
\hline & Euglycemia sample & & & & 3.50 & 5.05 & 0.63 \\
\hline \multirow[t]{4}{*}{12} & Hyperglycemia & 1,162 & 10.3 & 4.20 & 2.66 & 3.84 & 1.09 \\
\hline & Euglycemia & 574 & 7.8 & 2.95 & - & - & - \\
\hline & Hyperglycemia sample & & & & 4.02 & 5.80 & 0.51 \\
\hline & Euglycemia sample & & & & 3.77 & 5.44 & 0.54 \\
\hline
\end{tabular}


TABLE II (Continued)

\begin{tabular}{|c|c|c|c|c|c|c|c|}
\hline Patient & Period & $\begin{array}{l}\text { Glucose } \\
\text { brackets }\end{array}$ & $\mathrm{Hb} \mathrm{A} \mathrm{Alc}^{*}$ & $\begin{array}{c}\text { Fibrinogen } \\
\text { concentration } \downarrow\end{array}$ & $t^{t} / 2$ & $\begin{array}{l}\text { Fibrinogen } \\
\text { survival }\end{array}$ & $\begin{array}{c}\text { Fibrinogen } \\
\text { turnover }\end{array}$ \\
\hline & & & $\%$ & $m g / m l$ & $d$ & $d$ & $m g / m l / d$ \\
\hline \multirow[t]{4}{*}{13} & Hyperglycemia & 850 & 8.4 & 3.00 & 2.25 & 3.24 & 0.93 \\
\hline & Euglycemia & 520 & 8.1 & 3.80 & & & \\
\hline & Hyperglycemia sample & & & & 3.66 & 5.28 & 0.72 \\
\hline & Euglycemia sample & & & & - & - & - \\
\hline \multirow[t]{4}{*}{14} & Hyperglycemia & 1,426 & 7.5 & 2.80 & 1.83 & 2.64 & 1.06 \\
\hline & Euglycemia & 862 & 7.2 & 2.15 & & & - \\
\hline & Hyperglycemia sample & & & & 2.66 & 3.83 & 0.56 \\
\hline & Euglycemia sample & & & & 2.96 & 4.24 & 0.51 \\
\hline \multirow[t]{4}{*}{15} & Hyperglycemia & 1,276 & 8.4 & 4.20 & 2.19 & 3.15 & 1.33 \\
\hline & Euglycemia & 626 & 9.0 & 4.60 & & & \\
\hline & Hyperglycemia sample & & & & 3.01 & 4.33 & 1.06 \\
\hline & Euglycemia sample & & & & 2.87 & 4.14 & 1.11 \\
\hline
\end{tabular}

$* \mathrm{Hb} \mathrm{A}_{\mathrm{lc}}<5.0 \%$, normal value.

$\$$ Fibrinogen concentration $=1.60-3.50 \mathrm{mg} / \mathrm{ml}$, normal value.

However, the decrease in fibrinogen concentrations was not significant during the study with a mean value of $3.80 \pm 1.06 \mathrm{mg} / \mathrm{ml}$ during hyperglycemia and a mean value of $3.53 \pm 0.98 \mathrm{mg} / \mathrm{ml}$ during euglycemia.

Fibrinogen survival and turnover. Normal fibrinogen survival by the methods employed above is calculated to be $4.87 \mathrm{~d}(20)$. Fibrinogen survival in our patients was well below this normal level and increased significantly from a mean value of $3.45 \pm 0.57 \mathrm{~d}$ during hyperglycemia to a mean control value of $5.07 \pm 0.70 \mathrm{~d}(P<0.001)$ during the euglycemic period. Moreover, when studied in the euglycemic period, the survival of fibrinogen saved from the hyperglycemic period and fibrinogen prepared in the euglycemic period were virtually the same. Fig. 1 shows the fibrinogen survival curves obtained from one patient (patient 5) and illustrates the identical fibrinogen survival for the two samples in the euglycemic period. This result implies that an abnormal plasma environment rather than an abnormal fibrinogen molecule accounts for the reduced fibrinogen survival in "uncontrolled" diabetes. If an abnormal fibrinogen molecule modified via glycosylation was the cause for the observed decrease in survival during hyperglycemia one would expect divergent curves in the euglycemic phase with the fibrinogen molecule isolated during the hyperglycemic period disappearing at a more rapid rate than the sample isolated during the euglycemic period.

Fibrinogen turnover was reduced in all patients studied during hyperglycemia by an average of $34.4 \%$. The mean turnover value of $1.17 \pm 0.46 \mathrm{mg} / \mathrm{ml}$ per $\mathrm{d}$ improved to a mean value of $0.71 \pm 0.23 \mathrm{mg} / \mathrm{ml}$ per $\mathrm{d}$ $(P<0.02)$ during euglycemia. The reduced level of significance when comparing fibrinogen turnovers as opposed to fibrinogen survivals probably reflects the large variance in the plasma fibrinogen values.
Chromatographic and electrophoretic studies. To test whether the fibrinogen molecule is unchanged chemically as well as functionally during the hyperglycemic and euglycemic states of diabetes mellitus, chemical separation techniques were employed to compare the fibrinogen of diabetics with normal fibrinogen. Tracer amounts of labeled fibrinogen prepared for the survival studies during the hyperglycemic and euglycemic periods were mixed with unlabeled fibrinogen from a nondiabetic subject and examined for comparative elution characteristics with ion-exchange and gel-filtration techniques. The ${ }^{125}$ I-labeled sample

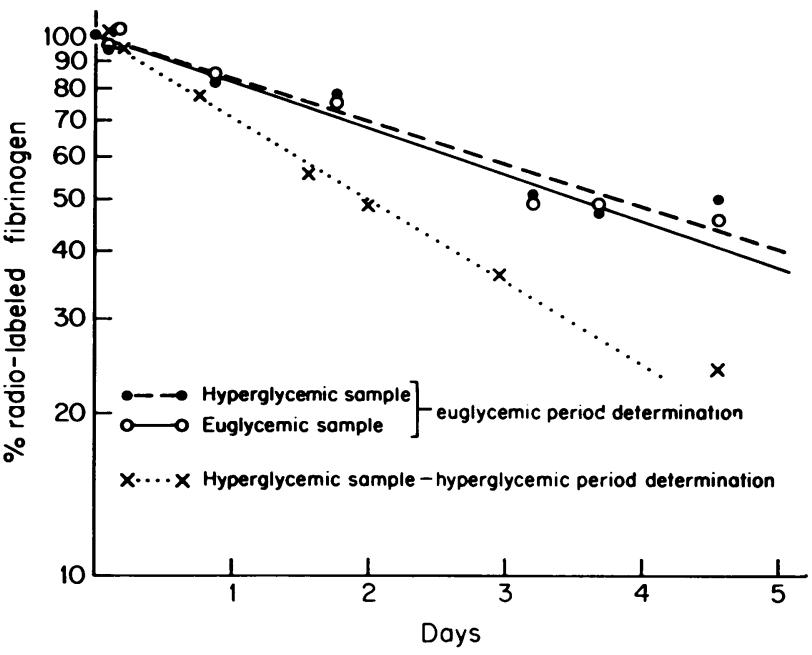

FIGURE 1 Fibrinogen survival curves from patient 5. In the hyperglycemic period fibrinogen survival was studied with autologous fibrinogen labeled with ${ }^{125}$ I. After carbohydrate control, fibrinogen survival was studied simultaneously with ${ }^{131}$ I-labeled fibrinogen purified during euglycemic period and ${ }^{125}$ I-labeled fibrinogen saved from the hyperglycemic period. 
from the hyperglycemic period and ${ }^{131}$-labeled sample from the euglycemic period coelute with the unlabeled fibrinogen from a nondiabetic subject. This result suggests that all these fibrinogen samples have similar charge. Similarly gel filtration chromatography showed no differences in molecular weight between diabetic hyperglycemic sample, euglycemic sample, and nondiabetic subject samples. Sodium dodecyl sulfatepolyacrylamide gel electrophoresis showed comigration of the major bands of samples isolated during periods of hyperglycemia and euglycemia from diabetic patients, nondiabetic fibrinogen purified in a similar manner and commercially available fibrinogen.

Pharmacological intervention. Four patients were placed on continuous heparin infusions and fibrinogen survival was repeated while the blood glucose remained at hyperglycemic level. Table III shows the results of these studies along with the mean partial thromboplastin time as monitored during the heparin infusion. Patients 7 and 15 had a mean partial thromboplastin time barely in the therapeutic range and had significant, although incomplete, normalization of fibrinogen survival from the hyperglycemic to the hyperglycemic(heparin) period. Patients 9 and 11 were maintained at the upper limits of the therapeutic range and each had normal fibrinogen survivals during the hyperglycemic(heparin) and euglycemic periods of the study.

The in vitro hyperaggregation of platelets in diabetes mellitus has been noted to be reversible when the blood glucose is normalized (2). It was conceivable that decreased fibrinogen survival during hyperglycemia could be a result of increased intravascular coagula- tion initiated or propagated by hyperaggregating platelets. Four patients were placed on aspirin and dipyridamole immediately after the initial study of fibrinogen survival and restudied during hyperglycemia with ${ }^{125}$ I-labeled sample. Inhibition of in vitro platelet aggregation was verified by complete elimination of the secondary wave of aggregation in response to $70 \mu \mathrm{M}$ epinephrine. An insignificant improvement in fibrinogen survival was seen during the hyperglycemic(aspirin and dipyrimadole) period, whereas a significant improvement occurred when the patients were brought under carbohydrate control (Table IV).

\section{DISCUSSION}

Vascular complications including atherosclerosis and microvascular disease now account for the major morbidity and mortality associated with diabetes mellitus (21). Recent attention to the interaction between the vascular system and coagulation factors implicates involvement of certain aspects of coagulation in the genesis of vascular lesions. Several observations suggest the existence of a hypercoagulable state in patients with diabetes, particularly in those patients with vascular disease. These observations include increased levels of fibrinogen and Factors V and VIII, and shortened plasma cephalin time $(22,23)$. Deposits of fibrin have been demonstrated in diabetic microvascular lesions by immunofluorescence (24). Thus, there appears to be evidence for a fibrinocoagulopathy concomitant with the development of diabetic vascular disease.

The present study confirms the existence of elevated

TABLE III

Results of Three Diabetic Patients Studied for Correction of Fibrinogen Survival with Intravenous Heparin Infusion

\begin{tabular}{clcccc}
\hline Patient & \multicolumn{1}{c}{ Period } & $\begin{array}{c}\text { Partial } \\
\text { thromboplastin } \\
\text { time }\end{array}$ & $\begin{array}{c}\text { Fibrinogen } \\
\text { concentration }\end{array}$ & $\begin{array}{c}\text { Fibrinogen } \\
\text { survival }\end{array}$ & $\begin{array}{c}\text { Fibrinogen } \\
\text { turnover }\end{array}$ \\
\hline \multirow{2}{*}{7} & $s$ & $m g / m l$ & $d$ & $m g / m l / d$ \\
& Hyperglycemic & 38 & 5.25 & 2.46 & 2.13 \\
& Hyperglycemic (heparin) & 58 & 5.25 & 4.09 & 1.28 \\
& Euglycemic & 34 & 2.25 & 4.68 & 0.48 \\
9 & Hyperglycemic & 40 & 3.76 & 3.19 & 1.18 \\
& Hyperglycemic (heparin) & 122 & 3.45 & 4.46 & 0.77 \\
& Euglycemic & 41 & 3.70 & 4.21 & 0.88 \\
& Hyperglycemic & 41 & 3.20 & 3.61 & 0.89 \\
& Hyperglycemic (heparin) & 128 & 3.00 & 5.51 & 0.54 \\
& Euglycemic & 44 & 3.20 & 5.17 & 0.62 \\
& Hyperglycemic & 39 & 4.20 & 3.15 & 1.33 \\
& Hyperglycemic (heparin) & 72 & 4.60 & 4.10 & 1.12 \\
& Euglycemic & 36 & 4.60 & 4.33 & 1.06 \\
\hline
\end{tabular}


TABLE IV

Results of Four Diabetic Patients Studied for Correction of Fibrinogen

Survival with Aspirin and Dipyrimadole

\begin{tabular}{|c|c|c|c|c|c|}
\hline Patient & Period & $\begin{array}{c}\text { Platelet } \\
\text { aggregation } \\
2^{\circ} \text { lag phase }\end{array}$ & $\begin{array}{l}\text { Fibrinogen } \\
\text { concentration }\end{array}$ & $\begin{array}{l}\text { Fibrinogen } \\
\text { survival }\end{array}$ & $\begin{array}{c}\text { Fibrinogen } \\
\text { turnover }\end{array}$ \\
\hline & & $s$ & & & \\
\hline \multirow[t]{3}{*}{6} & Hyperglycemic & 42 & 3.80 & 3.34 & 1.14 \\
\hline & Hyperglycemic (ASA and dipyr) & $>600$ & 4.20 & 3.49 & 1.20 \\
\hline & Euglycemic & 360 & 4.00 & 4.61 & 0.87 \\
\hline \multirow[t]{3}{*}{12} & Hyperglycemic & 38 & 4.20 & 3.81 & 1.09 \\
\hline & Hyperglycemic (ASA and dipyr) & $>600$ & 4.60 & 4.16 & 1.10 \\
\hline & Euglycemic & 60 & 2.95 & 5.80 & 0.51 \\
\hline \multirow[t]{3}{*}{13} & Hyperglycemic & 90 & 3.00 & 3.24 & 0.93 \\
\hline & Hyperglycemic (ASA and dipyr) & $>600$ & 3.25 & 2.88 & 1.13 \\
\hline & Euglycemic & 180 & 3.80 & 5.28 & 0.72 \\
\hline \multirow[t]{3}{*}{14} & Hyperglycemic & 66 & 2.80 & 2.64 & 1.06 \\
\hline & Hyperglycemic (ASA and dipyr) & $>600$ & 2.60 & 2.62 & 0.99 \\
\hline & Euglycemic & 400 & 2.15 & 3.83 & 0.56 \\
\hline
\end{tabular}

ASA, aspirin; dipyr, dipyrimadole.

fibrinogen levels in diabetic patients. A previous report showed that fibrinogen levels dropped significantly within $1 \mathrm{wk}$ after the initiation of insulin, tolbutamide, or phenformin therapy (25). Although there is a trend toward a decrease in fibrinogen levels in our data, we cannot confirm a significant decrease after control of diabetes as reflected by glucose brackets. Likewise, no significant changes in clotting function studies were appreciated during the hyperglycemia and euglycemia periods of the study.

Our study demonstrates that the survival of autologous radioiodinated fibrinogen is reduced in patients with adult-onset diabetes mellitus and varying degrees of vascular disease. During hyperglycemia native fibrinogen is consumed at an increased rate. The results are consistent with a previous report with $\left[{ }^{75} \mathrm{Se}\right.$ e]selenomethionine showing significantly shorter fibrinogen survival in diabetic as compared with normal subjects (5). We have extended this observation by demonstrating that increased fibrinogen consumption in uncontrolled diabetes is reversible when blood glucose values are optimized.

Blood and tissue proteins have been shown to be altered in diabetes by nonenzymatic glycosylation reactions thus altering their function or turnover $(26,27)$. In the case of hemoglobin, the conversion of hemoglobin $\mathrm{A}$ to hemoglobin $\mathrm{A}_{\mathrm{Ic}}$ proceeds by the nonenzymatic addition of glycosyl residues and the rate of adduct formation both in vitro and in vivo and is related to the ambient concentration of glucose (28). Results from the double-label experiments (simultaneous determination of survival of hyperglycemic period and euglycemic period fibrinogen molecules in the euglycemic period) indicate that an alteration in the fibrinogen molecule is not responsible for the reduced fibrinogen survival seen in hyperglycemic diabetic patients. If this were the case, one would expect divergent survival curves for the molecules during the hyperglycemic and euglycemic periods. Instead, the results are more consistent with an abnormal plasma and(or) vascular environment producing increased consumption of fibrinogen in the hyperglycemic diabetic state, which reverses toward normal in the euglycemic state.

The results of the double-label experiments do not preclude the possibility that fibrinogen is altered in uncontrolled diabetes. Examination of the samples purified for the survival studies by the techniques of gel filtration, iron exchange, and gel electrophoresis suggests no gross differences in charge or molecular weight or size between hyperglycemic period, euglycemic period, or fibrinogen purified from a normal individual. Furthermore, another report indicates no difference in glycogroups attached to fibrinogen between diabetic and normal subjects (29).

Four patients receiving anti-platelet agents during the hyperglycemic phase showed no significant improvement in fibrinogen survival. This result may be significant in light of recent reports that show in vitro hyperaggregation of platelets of patients with diabetes mellitus $(2,30,31)$. Although the in vivo significance of platelet hyperaggregation is obscure one might have expected correction of decreased fibrinogen survival by antiplatelet agents if the phenomenon is somehow linked to platelet hyperaggregation. 
Heparin is a potent anticoagulant that inhibits the action of thrombin as well as Factors XIa, IXa, Xa, and plasmin (32). Inhibition of these factors is believed to proceed by the potentiation of the protease inhibitor antithrombin III. Correction of reduced fibrinogen survival during hyperglycemia by heparin suggests involvement of one of these factors or antithrombin III in the fibrinogcoagulopathy of diabetes. It is generally accepted that the improvement of pathologically decreased fibrinogen survival by heparin is caused by interference with the action of thrombin on fibrinogen to form fibrin monomer $(20)$. Our results showing correction of decreased fibrinogen survival by heparin infusion support the concept that a hypercoagulable state exists in uncontrolled diabetes and implicates the action of thrombin or its antagonists.

The interval between the hyperglycemic study and euglycemic study varied between 1 and 9 wk with a mean of $2.5 \mathrm{wk}$. The abnormality is rapidly reversible because there is a higher correlation between fibrinogen survival and glucose brackets (a measure of short term control) than between fibrinogen survival and hemoglobin $A_{\text {Ic }}$ levels (Fig. 2). Because erythrocytes have a slow turnover, hemoglobin $A_{\mathrm{lc}}$ production represents an integrated measure of glycemia over weeks to months. Significant improvements in blood glucose levels do not lower hemoglobin $A_{\text {Ic }}$ values to a stable plateau for at least 3-4 wk (1). If modification of a protein other than fibrinogen is involved in the reversible hypercoagulable state, the protein must have a turnover measured in hours to days or the modification must be rapidly reversible.

Five of the eight patients with retinopathy had the shortest fibrinogen survival. This association is of interest in view of recent reports showing correlation of fibrinogen and Factor VIII (von Willibrand factor) antigen levels with retinopathy (33). It is not clear whether the clotting abnormalities in diabetes are

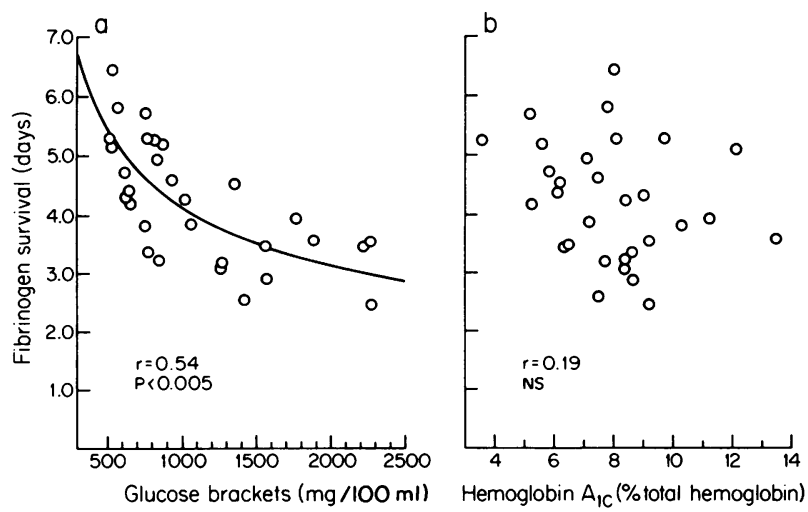

FIGURE 2 Correlation of fibrinogen survival with $(a)$ glucose brackets and $(b)$ hemoglobin $A_{\mathrm{Ic}}$. Regression line $(a)$ is the best fit and represents the expression $y=65.20 \chi^{-0.40}$. causative of, concomitant with, or a result of vascular disease. However, it seems unlikely that the reduced fibrinogen survival of hyperglycemia would be rapidly reversible during euglycemia if it were secondary to the presence of either macro- or microvascular disease. It should be emphasized that the present study was carried out with adult-onset diabetics all of whom presumably have some degree of vascular disease. Furthermore, studies involving juvenile-onset diabetes of recent onset and presumably without vascular disease are warranted to dissect the cause and effect relationship between coagulopathy and vascular disease in diabetes. If it is shown that the reversible hypercoagulable state participates in the genesis of vascular disease in patients with diabetes mellitus, it seems clear that more rigid control of blood glucose is indicated in preventing the onset of vascular complications. In addition, if the mechanism of the hypercoagulable state can be defined, then a more comprehensive prophylactic approach to vascular disease may be possible for these patients.

\section{ACKNOWLEDGMENTS}

We are indebted to Doctors Yale Nemerson and Vincent Dole for their assistance in the preparation of this manuscript. We are also indebted to Marge Peterson and Doris Lewis for their excellent technical assistance.

This work was supported by U. S. Public Health Service grants HL 20520 and AM 19655 and by a grant from Pfizer Inc.

\section{REFERENCES}

1. Peterson, C. M., and R. L. Jones. 1977. Minor hemoglobins, diabetic "control," and diseases of postsynthetic protein modification. Ann. Intern. Med. 87: 489-491.

2. Peterson, C. M., R. K. Koenig, R. L. Jones, E. T. Melvin, and M. L. Lehrman. 1977. Reversible hematologic sequelae of diabetes mellitus. Ann. Intern. Med. 86: 425-429.

3. Peterson, C. M., R. K. Koenig, R. L. Jones, C. D. Saudek, and A. Cerami. 1977. Correlation of serum triglyceride levels and hemoglobin $A_{I c}$ concentrations in diabetes mellitus. Diabetes. 26: 507-509.

4. Mustard, J. F., and M. A. Packham. 1975. The role of blood and platelets in atherosclerosis and the complications of atherosclerosis. Thromb. Diath. Haemorrh. 33: 44444456.

5. Ferguson, J. C., N. Mackay, J. A. D. Philip, and D. J. Sumner. 1975. Determination of platelet and fibrinogen half-life with [ ${ }^{75}$ Se]selenomethionine: Studies in normal and in diabetic subjects. Clin. Sci. Mol. Med. 49: 115120.

6. Winter, R. J., J. E. Wise, N. J. Stone, and O. C. Green. 1977. Correlations of 24 hour glucose profiles to standard parameters of diabetic control. Diabetes. 26(Suppl. 1): 364. (Abstr.)

7. Takeda, Y. 1966. Studies of the metabolism of fibrinogen in healthy men with autologous ${ }^{125}$ I labeled fibrinogen. J. Clin. Invest. 45: 103-111.

8. Clans, A. 1957. Gerinnungsphysiologische schnellmethode zur bestimmung des fibrinogens. Acta Haematol. (Basel). 17: 237-246. 
9. Tocantins, L. M., and L. A. Kazal, editors. 1964. Blood Coagulation, Hemorrhage and Thrombosis. Grune and Stratton, Inc., New York, 2nd edition. 103-107, 148150.

10. Weis, H. J. 1972. Platelet aggregation. In Hematology. W. J. Williams, E. Beutler, and A. J. Ershev, editors. McGraw-Hill Book Co., New York. 1415.

11. Levine, J., S. Morgenstern, and D. Vlastelizer. 1967. In Automation in Analytical Chemistry. 1967. Technicon Symposium. Medicoc, Inc., White Plains, N. Y.

12. Bucolo, G., and H. David. 1973. Quantitative determination of serum triglycerides by use of enzymes. Clin. Chem. 5: XIX 775.

13. Trivelli, L. A., H. N. Ranney, and H-T. Lai. 1971. Hemoglobin components in patients with diabetes mellitus. $N$. Engl. J. Med. 284: 353-357.

14. Javid, J., P. K. Pettis, R. J. Koenig, and A. Cerami. 1978. Immunologic characterization and quantification of hemoglobin $\mathrm{A}_{\mathrm{Ic}}$. Br. J. Haematol. 38: 329-337.

15. Finlayson, J. S., and M. W. Mosesson. 1963. Heterogeneity of human fibrinogen. Biochemistry. 2: 42-46.

16. Berg, W., and K. Korsan-Bengtsen. 1963. Separation of human fibrinogen and plasminogen by means of gel filtration. Thromb. Diath. Haemorrh. 9: 151-154.

17. Laemmli, U. K. 1970. Cleavage of structural proteins during the assembly of the head of bacteriophage $T_{4}$. Nature (Lond.). 242: 680-685.

18. Kazal, L. A., S. Amsel, O. P. Miller, and L. M. Tocantins. 1963. The preparation and some properties of fibrinogen precipitated from human plasma by glycine. Proc. Soc. Exp. Biol. Med. 113: 989-994.

19. Hamburg, M. 1974. Basic Statistics. Harcourt Brace Jovanovich, Inc., New York. 166-200.

20. Reeve, E. B., and J. J. Franks. 1974. Fibrinogen synthesis, distribution and degradation. Semin. Thromb. Hemostasis. 1: 129-183.

21. Bradley, R. F. 1959. Cardiovascular disease. In Joslin's Diabetes Mellitus. A. Marble, P. White, R. Bradley, and L. P. Krall, editors. Lea \& Febiger, Philadelphia. 417477.
22. Egebert, O. 1963. The blood coagulability in diabetic patients. J. Clin. Lab. Med. 15: 833-838.

23. Mayne, E. E., J. M. Bridges, and J. A. Weaver. 1970. Platelet adhesiveness, plasma fibrinogen and factor VIII level in diabetes mellitus. Diabetologia. 6: 436-440.

24. Bannerjee, R. N., A. L. Sahni, and V. Kumar. 1973. Fibrinocoagulopathy in maturity onset diabetes mellitus and atherosclerosis. Thromb. Diath. Haemorrh. 30: 123-132.

25. Bedi, H. K., B. R., Vyas, B. S. Bomb, M. L., and T. Bedi. 1977. Fibrinogen content and fribinolytic activity of blood in diabetics before and after antidiabetic drugs. J. Assoc. Physicians. India 25: 181-185.

26. Bunn, H. F., D. N. Haney, S. Kamin, K. H. Gabbay, and P. M. Gallop. 1976. The biosynthesis of human hemoglobin $\mathrm{A}_{\mathrm{Ic}}$ : Slow glycosylation of hemoglobin in vivo. J. Clin. Invest. 57: 1652-1659.

27. Stevens, V. J., C. A. Rouzer, V. M. Monnier, and A. Cerami. 1978. Diabetic cataract formation: The potential role of glycosylation of lens crystallins. Proc. Natl. Acad. Sci. U. S. A. 75: 2918-2922.

28. Stevens, V. S., H. Vlassara, A. Abati, and A. Cerami. 1977. Nonenzymatic glycosylation of hemoglobin. J. Biol. Chem. 252: 2998-3002.

29. Palini, M., I. Lipinski, V. Garewick, and E. E. Grodon. 1977. Low molecular weight fibrinogen in diabetes mellitus. Diabetes. 26(Suppl.): 369. (Abstr.)

30. Heath, H., W. D. Brigden, and J. V. Canever, J. Pollock, P. R. Hunter, J. Kelsey, and A. Bloom. 1971. Platelet adhesiveness and aggregation in relation to diabetic retinopathy. Diabetologia. 7: 309-315.

31. Kwaan, H. C., J. A. Colwell, S. Cruz, N. Suwanwela, and J. G. Dobbie. 1972. Increased platelet aggregation in diabetes mellitus. J. Lab. Clin. Med. 80: 236-246.

32. Hirsh, J., and A. S. Gallus. 1977. Heparin. In Recent Advances in Hematology. A. V. Hoffbrand, M. C. Brain, and J. Hirsch, editors. Churchill Livingston, Edingburgh. 452-466.

33. Coller, B. S., R. N. Frank, R. C. Milton, and H. R. Gralnick. 1978. Plasma cofactors of platelet function: Correlation with diabetic retinopathy and hemoglobins $\mathrm{A}_{\mathrm{la}-\mathrm{c}}$. Ann. Intern. Med. 88: 311-316. 